\title{
Presidential address: Annual Academic Sessions of College of Pathologists of Sri Lanka 2014
}

\section{Pathologist's role in non-communicable diseases}

DOI: http://doi.org/10.4038/jdp.v11i2.7703

\section{Epidemiology \\ With the control of communicable diseases by improved health care facilities, non communicable diseases (NCD) have surpassed communicable diseases in morbidity and mortality in Sri Lanka. Among these, cardiovascular diseases have the highest mortality with respiratory diseases, trauma, poisoning, diabetes, kidney, liver diseases followed by cancers showing an upward trend. Although there is an increase in incidence of cancer from 1990 to 2007, cancer related deaths remain static due to early detection and better treatment modalities provided by health care services. However, malignancies had the highest mortality in 2009 followed by deaths related to cardiovascular causes.}

Cancer is a major component of the non communicable disease burden in Sri Lanka with an increase in incidence of 31.6 in 1995 to 71.6 in 2007 per 100,000 population. Cancer detection and management constitute a major component of the health care system in Sri Lanka. Therefore pathologists play a crucial role in diagnosis of malignancy. Among the Sri Lankan males, the common sites of malignancy are lip, oral cavity and pharynx, trachea, bronchus, lung and oesophagus, followed by colorectum, lymphoma, leukemia, larynx, prostate and stomach; commonest three cancer sites among females are breast, cervix uteri and thyroid followed by oesophagus, ovary, colorectum, lip, oral cavity, pharynx, leukemia, uterus and lymphoma. The five most common malignancies without gender consideration are breast, lip and oral cavity, pharynx, cervix uteri and oesophagus. Leukaemias, lymphomas and brain tumours are the common malignancies among children (Source: National Cancer Control Programme data).

The role of the histopathologist in malignancy is for prevention, diagnosis and prognostication

Breast cancer is the most common cancer affecting females worldwide including Sri Lanka. Although Sri Lanka does not have a formal screening programme by mammography, the National Cancer Control Programme (NCCP) has published guidelines for primary care doctors for early detection and management of breast symptoms. Histopathologists report on tru cut biopsies of radiologically detected breast lesions and hook wire guided biopsies. Although fine needle aspiration has gone out of fashion in many countries in Sri Lanka fine needle aspiration is still commonly performed for palpable lumps. Core needle biopsy is done for atypical cytology/inconclusive category (C3), patients presenting with non-palpable breast lesions, advanced tumours, prior to surgery and neo-adjuvant therapy in breast cancer. 
Breast cancer prognosis depends on histopathological grade, stage and prognostic markers. Biomarkers analyzed are oestrogen and progesterone receptors, Ki67 proliferative index and human epidermal growth factor receptor- Her - 2. Targeted therapy is given for hormone receptor and Her -2 positive patients. These are detected by immunohistochemistry and fluorescence in situ hybridization methods.

Cervical cancer is the second commonest cancer affecting Sri Lankan females with an incidence of 6.6 per 100,000 population. In 2009 there were 1540 new cases detected according to WHO statistics. Cervical cancer has a long pre-invasive stage which is easily detected by cervical smear testing. At this stage the treatment is simple and curative. Primary prevention of human papilloma virus infection is by 3 doses of Cervirax vaccine given 6 months apart at 10 years of age. However, this is not practiced in Sri Lanka at present. Secondary prevention is by cervical Pap smear screening and treating the pre invasive stage.

\section{Cervical screening programme}

The policies determine the maximal effectiveness; implementation determines the actual effectiveness of the programme. Success of cervical screening has been demonstrated in the USA, where before the Pap screening programme, the incidence of cervical cancer was 50 per 100,000 population and after implementation of the programme the incidence was reduced to 8 per 100,000 population. In Sri Lanka the effectiveness of the programme has not yet been assessed. Services offered by the well woman clinics in Sri Lanka are screening of females above 35 years for NCDs like diabetes, hypertension, detection of breast abnormalities and referral for mammography and cervical cancer screening.

Cervical Pap screening programme in Sri Lanka was introduced in 1996 and with UNFPA funding from 1997 it was expanded to most of the provinces. The working group includes the Director General of Health and the focal point is the Family Health Bureau. Stakeholders of the programme are the National Cancer Control Programme, College of Pathologists of Sri Lanka, Sri Lanka College of Obstetricians and Gynaecologists \& Laboratory Services. Establishment of cervical screening laboratories is coordinated by the College of Pathologists of Sri Lanka. Laboratories manned by histopathologists are included in the programme. Medical laboratory technologists are trained as cytoscreeners. Training and examinations of cytoscreeners are conducted by the College of Pathologists. Abnormalities detected by cytoscreeners are assessed and diagnosed by cytopathologists enabling patient benefits from early diagnosis and treatment of the pre-invasive stage of cervical cancer. Interpretation of smears with follow up and treatment is done according to the National guidelines. New developments in screening are liquid based cytology, computer assisted screening of smears and HPV DNA testing using hybrid capture, PCR and FISH. These new developments have not yet reached the shores of Sri Lanka.

Cancers of the lip, oral cavity and pharynx are the commonest malignancies affecting Sri Lankan males and the $7^{\text {th }}$ commonest cause in females. More than $90 \%$ of the malignancies in this region are squamous cell carcinomas. Oral premalignant lesions except lichen planus are associated with habits of betel and arecanut chewing, smoking, snuff dipping and alcohol intake and prevention is by avoiding the risk factors. Sri Lanka has established non smoking zones in public areas. If we could ban 
betel and arecanut chewing in public places we could reduce the incidence of oral, pharyngeal, laryngeal, lung, oesophgeal and other cancers significantly.

A Risk factor model has been developed and published by the Ministry of Health to identify individuals who are at risk of developing pre-malignant oral lesions and oral cancer. Individuals with a total score of 12 and above are targeted for oral screening. Active screening of high risk communities and opportunistic screening in patients presenting to dental clinics are managed according to the national guidelines. The National Health Survey done in 2002 and 2003 revealed that the prevalence of oral pre malignant lesions are $3.4 \%$ in the age groups of 34 to 44 and 65 to 75 years respectively. For central Sri Lanka the prevalence was $4.2 \%$ in the general population and $6.7 \%$ among the estate workers. Sabaragamuwa province had a prevalence of $11 \%$ in the rural and estate sector. The National Cancer Control Programme has published guidelines for detection of oral potentially premalignant lesions, namely, submucous fibrosis, leukoplakia, erythroplakia and lichen planus. These lesions progress to dysplasia and squamous cell carcinoma. The role of the pathologist is to diagnose premalignant and malignant lesions enabling the patient to have the appropriate treatment.

Thyroid cancers are the 3rd commonest cancer affecting Sri Lankan females. Fine needle aspiration is essential for diagnosis in solitary nodules of the thyroid. FNA interpretation to the referring clinician should be succinct, unambiguous and clinically helpful in the management of the patient. The Bethesda system for reporting thyroid cytology meets these demands. The diagnostic categories are non diagnostic, benign, atypia of undetermined significance, follicular neoplasms, suspicious of malignancy and malignant. The Bethesda system of reporting with the implied risk of malignancy and recommended management has greatly reduced surgery done for non malignant thyroid lesions.

Trachea, bronchus and lung cancers are the second commonest cancer affecting Sri Lankan males. Bronchial brush, wash cytology and biopsies are diagnosed by the pathologist. Squamous, adeno and small cell carcinomas are the commonest types seen in Sri Lanka.

Most prostate cancers in Sri Lanka are high grade. Screening for prostate cancer should be done in patients with obstructive lower urinary tract symptoms, serum PSA of $>4$ $\mathrm{ng} / \mathrm{ml}$, and if the digital rectal examination is suspicious. Six quadrant trans-rectal ultrasound guided core biopsies are done and histologically looked for prostatic intraepithelial neoplasia or adenocarcinoma. Elevated serum Prostate Specific Antigen is seen in prostatic carcinoma and false positive levels are seen in granulomatous prostatitis, prolonged indwelling catheter and in chronic prostatitis. Gleason grading system, which is a prognostic indicator, is done by the histopathologist.

Colonic cancer prevention is by life style modification mainly by consuming high fiber diet rich in fruits and vegetables and low in fat and red meat with plenty of exercise and weight reduction. Screening colonoscopy is recommended in patients with altered bowel habits or family history of colonic cancer. Early diagnosis is by screening colonoscopy, polypectomy and colectomy. Diagnosis is by histopathology of the biopsy. Prognosis depends on Dukes and TNM Stage at the time of surgery.

In conclusion, for cervical and oral cancer national screening programmes are available at present, increasing awareness among the 
general population regarding early symptoms of malignancy and seeking medical advice early. The National Cancer Control Programme is working towards achieving this goal. We recommend similar screening programmes for breast, colorectal and prostate malignancies. Since pathologists play a major role in early diagnosis, diagnosis and prognostication of malignancy, we recommend an increase of training positions and cadre posts in stations with high workload and cancer referral centres. Establishment of regional cluster systems with a central referral centre of excellence would facilitate obtaining second opinion as histopathologists have not subspecialized. Such referral centres should provide specialized diagnostic modalities like immunohistochemistry, electron microscopy, FISH , PCR and cytogenetics for tumours.

\section{Dr M M A Jayawickrama}

President, College of Pathologists of Sri Lanka $9^{\text {th }}$ September 2014 Hotel Ramada, Colombo, Sri Lanka 